\title{
ON RIGHT UNIPOTENT SEMIGROUPS II
}

\author{
by P. S. VENKATESAN
}

(Received 3 August, 1976)

We describe two congruences $\alpha$ and $\gamma$ contained in $\mathscr{L}$ on an arbitrary orthodox semigroup. Let $S$ be a right unipotent semigroup. We show that (i) $\alpha$ is an inverse semigroup congruence and $\gamma$ is the finest fundamental inverse semigroup congruence on $S$, (ii) $S$ is a union of groups if and only if $\gamma=\mathscr{L}$ on $S$ and (iii) $S$ is a band of groups if and only if $\alpha=\mathscr{L}$ on $S$.

Let $A$ be a regular semigroup and let $x \in A$. Throughout the paper $V(x)$ stands for the set of inverses of $x$, and $E(A)$ for the set of idempotents of $A$. $A$ is said to be an orthodox semigroup if $E(A)$ is a subsemigroup of $A$. If $A$ is an orthodox semigroup then $V(b) V(a) \subseteq V(a b)$ for all $a, b$ in $A$ [9]. For the general terminology and notation, the reader is referred to [2].

The author wishes to thank the referee for his suggestions and remarks.

1. The congruences $\alpha, \beta, \gamma, \delta$ and $\mu$. Let $S$ be an orthodox semigroup and let $E=E(S)$. The greatest idempotent-separating congruence $\mu$ on $S$ (Howie [5, Theorem VI. 1.17], Meakin [7]) is given by the rule:

$(x, y) \in \mu=\mu(S) \Leftrightarrow x^{\prime} e x=y^{\prime} e y$

and $x e x^{\prime}=y e y^{\prime}$ for all $e \in E$ and for some $x^{\prime} \in V(x)$ and $y^{\prime} \in V(y)$.

We define the binary relations $\alpha$ and $\gamma$ on $S$ thus:

$(x, y) \in \alpha=\alpha(S) \Leftrightarrow x^{\prime} e x=y^{\prime} e y$ for all $e \in E$ and for some $x^{\prime} \in V(x), y^{\prime} \in V(y)$;

$(x, y) \in \gamma=\gamma(S) \Leftrightarrow x^{\prime} e x y^{\prime} e y=x^{\prime} e x$ and

$$
y^{\prime} e y x^{\prime} e x=y^{\prime} e y \text { for all } e \in E \text { and for some } x^{\prime} \in V(x), y^{\prime} \in V(y) \text {. }
$$

The proof of Theorem 1 below is like Meakin's (loc. cit.) derivation of $\mu$ for $S$.

THEOREM 1. Let $S$ be an orthodox semigroup and let $E=E(S)$. Then

(1) $\alpha$ and $\gamma$ are congruences on $S$,

(2) $\gamma$ is the greatest congruence contained in $\mathscr{L}$ on $S$.

Proof. Clearly $\alpha$ is reflexive and symmetric. Let $(x, y) \in \alpha$. Then there exist $x^{\prime} \in V(x)$ and $y^{\prime} \in V(y)$ such that $x^{\prime} e x=y^{\prime} e y$ for all $e \in E$. Let $x^{\prime \prime} \in V(x)$ and $y^{\prime \prime} \in V(y)$. Taking in turn $e=x x^{\prime \prime}$ and $e=y y^{\prime \prime}$, we have $x^{\prime} x=y^{\prime} x x^{\prime \prime} y$ and $y^{\prime} y=x^{\prime} y y^{\prime \prime} x$. Therefore $x^{\prime} x=x^{\prime} x y^{\prime} y=$ $y^{\prime} y$. If $(y, z) \in \alpha$ then $y^{*} e y=z^{\prime} e z$ for all $e \in E$ and some $y^{*} \in V(y), z^{\prime} \in V(z)$. So $y^{*} y=$ $z^{\prime} z=z^{\prime} y y^{\prime} z$. Set $p=y^{*} y x^{\prime}$ and $q=z^{\prime} y y^{\prime}$. Then $p x p=y^{*}\left(y x^{\prime} x y^{*} y x^{\prime}\right)=y^{*} y x^{\prime}=p$ and $x p x=$ $x y^{*} y\left(x^{\prime} x\right)=x y^{*} y=\left(x y^{\prime} y\right) y^{*} y=x y^{\prime} y=x$. Therefore $p \in V(x)$. Similarly $q \in V(z)$. Now, for all $e \in E$, we have pex $=y^{*} y\left(x^{\prime} e x\right)=y^{*} y\left(y^{\prime} e y\right)=y^{*}\left(y y^{\prime} e\right) y=z^{\prime}\left(y y^{\prime} e\right) z=q e z$, giving $(x, z) \in \alpha$. So $\alpha$ is transitive and hence an equivalence relation on $S$.

Glasgow Math. J. 19 (1978) 63-68. 
Let $a \in S$ and $a^{\prime} \in V(a)$. Then for all $e \in E$ we have $a^{\prime}\left(x^{\prime} e x\right) a=a^{\prime}\left(y^{\prime} e y\right) a$ and $x^{\prime}\left(a^{\prime} e a\right) x=y^{\prime}\left(a^{\prime} e a\right) y$. Since $a^{\prime} e a \in E[9]$, we get that $\alpha$ is a congruence on $S$.

We now consider $\gamma$, which is clearly reflexive and symmetric. Let $(x, y) \in \gamma$. Then $x^{\prime} e x y^{\prime} e y=x^{\prime} e x$ and $y^{\prime} e y x^{\prime} e x=y^{\prime} e y$ for all $e \in E$ and some $x^{\prime} \in V(x), y^{\prime} \in V(y)$. If $(y, z) \in \gamma$ then there exist inverses $y^{*}$ of $y$ and $z^{\prime}$ of $z$ such that $y^{*} e y z^{\prime} e z=y^{*} e y$ and $z^{\prime} e z y^{*} e y=$ $z^{\prime} e z$. Since $S$ is orthodox, we have $y^{\prime} e y=y^{\prime}\left(e y y^{*} e y\right)$. Therefore $x^{\prime} e x z^{\prime} e z=x^{\prime} e x y^{\prime} e y z^{\prime} e z=$ $x^{\prime} e x\left(y^{\prime} e y y^{*} e y\right) z^{\prime} e z=x^{\prime} e x\left(y^{\prime} e y y^{*} e y\right)=x^{\prime} e x y^{\prime} e y=x^{\prime} e x$. Similarly, $z^{\prime} e z x^{\prime} e x=z^{\prime} e z$. So $(x, z) \in \gamma$, and $\gamma$ is an equivalence relation.

Let $a \in S$ and $a^{\prime} \in V(a)$. Then since $(x, y) \in \gamma$, for all $e \in E$ we have $x^{\prime}\left(a^{\prime} e a\right) x y^{\prime}\left(a^{\prime} e a\right) y=x^{\prime}\left(a^{\prime} e a\right) x$ and $y^{\prime}\left(a^{\prime} e a\right) y x^{\prime}\left(a^{\prime} e a\right) x=y^{\prime}\left(a^{\prime} e a\right) y$. Therefore $(a x, a y) \in \gamma$. Further $a^{\prime} x^{\prime}$ exa $a^{\prime} y^{\prime}$ eya $=a^{\prime}\left(x^{\prime}\right.$ exy'ey $) a a^{\prime} y^{\prime}$ eya $=a^{\prime} x^{\prime}$ exy $($ eya $)\left(a^{\prime} y^{\prime} e\right)($ eya $a)=a^{\prime} x^{\prime}$ exy' $($ eya $)=$ $a^{\prime}\left(x^{\prime} e x y^{\prime} e y\right) a=a^{\prime} x^{\prime} e x a$. Similarly, $a^{\prime} y^{\prime} e y a a^{\prime} x^{\prime} e x a=a^{\prime} y^{\prime} e y a$. Thus $(x a, y a) \in \gamma$, and $\gamma$ is a congruence on $S$.

As for the other part, if $(x, y) \in \gamma$, taking $e=x^{\prime} x$ we have $x^{\prime} x y^{\prime} x x^{\prime} y=x^{\prime} x$. So $x y^{\prime} x x^{\prime} y=x$ and hence $x y^{\prime} y=x$. Similarly $y x^{\prime} x=y$. Therefore $(x, y) \in \mathscr{L}$ and $y \subseteq \mathscr{L}$.

Now let $\eta$ be a congruence on $S$ such that $\eta \subseteq \mathscr{L}$. Let $(x, y) \in \eta$. Then, for all $e \in E$, we have $(e x, e y) \in \mathscr{L}$. So for all inverses $p$ of $e x$ and $q$ of $e y$, we have pexqey $=p e x$ and qeypex $=q e y$. Taking $p=x^{*} e$ and $q=y^{*} e$, where $x^{*}$ and $y^{*}$ are inverses of $x$ and $y$ respectively, we get that $(x, y) \in \gamma$ and hence that $\eta \subseteq \gamma$. This completes the proof of the theorem.

COROLlaRY. If $\mathscr{L}$ is a congruence on an orthodox semigroup, then $\gamma=\mathscr{L}$.

Remark. Let $S$ be an orthodox semigroup and let $x, y \in S$. Then, since $\gamma \subseteq \mathscr{L}$, we have $(x, y) \in \gamma$ if and only if $x^{\prime} e x y^{\prime} e y=x^{\prime} e x$ and $y^{\prime} e y x^{\prime} e x=y^{\prime} e y$ for all $e \in E(S), x^{\prime} \in V(x)$ and $y^{\prime} \in V(y)$.

Remark. Let $S$ be an orthodox semigroup and let $E=E(S)$. Define the binary relations $\beta$ and $\delta$ on $S$ thus:

$(x, y) \in \beta \Leftrightarrow x e x^{\prime}=y e y^{\prime}$ for all $e \in E$ and some $x^{\prime} \in V(x), y^{\prime} \in V(y)$;

$(x, y) \in \delta \Leftrightarrow x e x^{\prime} y e y^{\prime}=y e y^{\prime}$ and $y e y^{\prime} x e x^{\prime}=x e x^{\prime}$ for all $e \in E$ and some $x^{\prime} \in V(x), y^{\prime} \in V(y)$.

Then $\beta$ and $\delta$ are congruences on $S$, and $\delta$ is the greatest congruence contained in $\mathscr{R}$ on $S$. Trivially $\beta \subseteq \delta(\alpha \subseteq \gamma)$, and it follows from the definitions of $\alpha, \beta, \gamma, \delta$ and $\mu$ that $\mu=\alpha \cap \beta=\gamma \cap \delta$.

A semigroup is said to be fundamental if the only congruence on it contained in $\mathscr{H}$ is the trivial congruence. Since $\mu$ is the greatest congruence on $S$ contained in $\mathscr{H}$, it follows that $S$ is fundamental if and only if $\mu$ is the trivial congruence on $S$. We omit the proof of the following result which is similar to the one known for inverse semigroups (Howie [4], Munn [8]).

Lemma 1. Let $S$ be an orthodox semigroup. Then $S / \mu$ is a fundamental orthodox semigroup and the band of idempotents of $S / \mu$ is isomorphic to $E(S)$.

Theorem 2 below shows how certain homomorphic images of $S$ are related. 
Theorem 2. Let $S$ be an orthodox semigroup. Write $L=S / \mu, M=S / \alpha, N=S / \gamma$, $\alpha^{\prime}=\alpha(L), \gamma^{\prime}=\gamma(L)$ and $\gamma^{\prime \prime}=\gamma(M)$. Then

(1) $L / \alpha^{\prime}$ is isomorphic to $M$,

(2) $L / \gamma^{\prime}$ and $M / \gamma^{\prime \prime}$ are isomorphic to $N$.

Proof. By [2, Theorem 1.6], the mappings $\theta: L \rightarrow M, \phi: L \rightarrow N$ and $\psi: M \rightarrow N$ defined by $(a \mu) \theta=a \alpha,(a \mu) \phi=a \gamma$ and $(x \alpha) \psi=x \gamma(a, x \in S)$, are surjective homomorphisms. Let $a, b \in S$. Then, since every idempotent of $M$ is the image of an idempotent of $L$ [6], we have $(a, b) \in \alpha$ if and only if $(a \mu, b \mu) \in \alpha^{\prime}$. So $\theta \circ \theta^{-1}=\alpha^{\prime}$, proving (1). Since $(a, b) \in \gamma$ if and only if $(a \mu, b \mu) \in \gamma^{\prime}$, we get the first part of (2). If $x, y \in S$, then $(x, y) \in \gamma$ if and only if $(x \alpha, y \alpha) \in \gamma^{\prime \prime}$, proving the other part of (2).

Remark. Write $T=S / \beta, \beta^{\prime}=\beta(L)$ and $\delta^{\prime}=\delta(T)$. Then proceeding as above we get $L / \beta^{\prime}$ isomorphic to $T$, and $T / \delta^{\prime}$ isomorphic to $S / \delta$.

2. Right unipotent semigroups. Let $A$ be an orthodox semigroup and let $E=E(A)$. The finest inverse semigroup congruence $\sigma$ on $A$ (Hall [3], Yamada [14]) is given by the rule: $(x, y) \in \sigma$ if and only if $V(x)=V(y)$.

$A$ is said to be a right (left) unipotent semigroup if every principal right (left) ideal of $A$ has a unique idempotent generator. Let $S$ be a right unipotent semigroup and let $a \in S$. Then (i) $f e f=f e$ for any two idempotents $e, f$ of $S$, and (ii) $a a^{\prime}=a a^{*}$ for any $a^{\prime}, a^{*}$ in $V(a)$ [11]. Further, $\beta$ is the greatest idempotent-separating congruence on $S$ [12]. Since $\beta=\delta$ follows from the definition of $S$, we have $\mu=\beta=\delta$ on $S$. That $\mu=\alpha=\beta$ on an inverse semigroup is well known (Howie [4]).

Theorem 3. Let $S$ be an orthodox semigroup and let $E=E(S)$. Then the following statements are equivalent:

(A) $S$ is a right unipotent semigroup;

(B) $S / \alpha$ is an inverse semigroup;

(C) $S / \gamma$ is an inverse semigroup.

Proof. Assume (A). Let $g, h \in E$. Then, for all $e \in E$, we have ghehg = ghe $=$ ghegh [11]. This, since $V(g h)=V(h g)$, implies that $(h g, g h) \in \alpha$. Since every idempotent of $S / \alpha$ is the image of an idempotent of $S[6]$, we get (B).

(B) implies (C) since $S / \gamma$ is a homomorphic image of $S / \alpha[2, p .17]$. That (C) implies (A) follows from [13]. For a direct proof, assume (C). Let $g, h \in E$. Then, since $\gamma$ is contained in $\mathscr{L}$, we have $(g h, h g) \in \mathscr{L}$. Therefore $g h g=g h$, proving (A) [11].

TheOREm 4. Let $S$ be a right unipotent semigroup and let $E=E(S)$. Write $P=S / \sigma$ and $\mu^{\prime}=\mu(P)$. Then

(1) $P / \mu^{\prime}$ is isomorphic to $S / \gamma$,

(2) $\gamma$ is the finest fundamental inverse semigroup congruence on $S$.

Proof. By Theorem 3 we have $\sigma \subseteq \gamma$. So, proceeding on the lines of the proof of Theorem 2, we get (1). From this result and Lemma 1 it follows that $S / \gamma$ is a fundamental inverse semigroup. Let $\rho$ be a congruence on $S$ such that $S / \rho$ is a fundamental inverse 
semigroup. Let $x, y \in S$ be such that $(x, y) \in \gamma$. Then, since $S / \rho$ is inverse, we have $(x \rho, y \rho) \in \mu$ on $S / \rho$. This, since $S / \rho$ is fundamental, implies that $(x, y) \in \rho$ and hence that $\gamma \subseteq \rho$.

Corollary (to the proof of (1)). The semilattice $E(S / \sigma)$ is isomorphic to the semilattice $E(S / \gamma)$.

3. Unions of groups. In this section we consider right unipotent semigroups which are unions of groups.

Let $S$ be a right unipotent semigroup, let $E=E(S)$ and let $x \in S$. Let the symbols $(P x)$ and $(Q x)$ denote statements as follows:

$(P x)$ exe $=e x$ and $e x^{\prime} e=e x^{\prime}$ for all $e \in E$ and for some $x^{\prime} \in V(x)$.

$(Q x) x e x^{\prime}=x x^{\prime} e$ for all $e \in E$ and $x^{\prime} \in V(x)$.

Then (i) $(Q x)$ implies $(P x)$ for any $x$ in $S$, (ii) $S$ is a union of groups if and only if $(P x)$ is satisfied for all $x$ in $S$ and (iii) $S / \beta$ is isomorphic to $E$ if and only if $(Q x)$ is satisfied for all $x$ in $S[12]$.

Let $S$ be a right unipotent semigroup which is a union of groups. Then Green's relations on $S$ are related thus: $\mathscr{g}=\mathscr{D}=\mathscr{L}$ and $\mathscr{R}=\mathscr{H}$ [10].

THEOREM 5. Let $S$ be a right unipotent semigroup and let $E=E(S)$. Then the following statements are equivalent:

(A) $S$ is a union of groups;

(B) $S / \sigma$ is a semilattice $Y$ of groups, where $Y$ is isomorphic to $E(S / \sigma)$;

(C) $S / \gamma$ is a semilattice;

(D) $\gamma=\mathscr{L}$ (equivalently, $\mathscr{L}$ is a congruence on $S$ );

(E) $S$ is a semilattice $Z$ of left groups, where $Z$ is isomorphic to $S / \gamma$.

Proof. That (A) implies (B) is well known [2, pp. 126-129]. Assume (B). Let $x \in S$, $x^{\prime} \in V(x)$ and $e \in E$. Since the idempotents of $S / \sigma$ are in the centre of $S / \sigma$ [2, p. 127], we have $(e x) \sigma=(x e) \sigma$; that is, $V(e x)=V(x e)$. Now both $x^{\prime} e$ and $e x^{\prime}$ are inverses of $e x$. This, since $S$ is right unipotent, implies that $e x e x^{\prime}=e x x^{\prime}$. Similarly $e x^{\prime} e x=e x^{\prime} x$. Therefore

and

$$
\left(x^{\prime} e x\right)\left(x^{\prime} \operatorname{ex}^{\prime} x\right)=x^{\prime}\left(e x e x^{\prime}\right) x=x^{\prime}\left(e x x^{\prime}\right) x=x^{\prime} e x
$$

$$
\left(x^{\prime} x e x^{\prime} x\right)\left(x^{\prime} e x\right)=x^{\prime} x\left(e x^{\prime} e x\right)=x^{\prime} x e x^{\prime} x .
$$

So $\left(x, x^{\prime} x\right) \in \gamma$ and hence, by Theorem $3, S / \gamma$ is a semilattice, proving (C).

Assume (C). We first prove that $(P x)$ is satisfied for all $x \in S$. Let $x \in S, x^{\prime} \in V(x)$ and $e \in E$. Then $\left(x^{\prime} e, e x^{\prime}\right) \in \gamma$. This, since $\gamma \subseteq \mathscr{L}$, implies that $\left(e x x^{\prime} e\right)\left(x e x^{\prime}\right)=e x x^{\prime} e$. Therefore, since $S$ is right unipotent, we have $e x e x^{\prime}=e x x^{\prime}$ and hence

$$
\text { exe }=e x\left(x^{\prime} x e\right)=e x\left(x^{\prime} x e x^{\prime} x\right)=\left(e x e x^{\prime}\right) x=\left(e x x^{\prime}\right) x=e x .
$$

Similarly $e x^{\prime} e=e x^{\prime}$. So $(P x)$ is satisfied for all $x \in S$.

Now let $(x, y) \in \mathscr{L}$. If $x^{\prime} \in V(x)$ and $y^{\prime} \in V(y)$ then $x y^{\prime} y=x$ and $y x^{\prime} x=y$. So, for all $e \in E$, using $\left(P x y^{\prime}\right)$, we have $x^{\prime} e x y^{\prime} e y=x^{\prime}\left(e x y^{\prime} e\right) y=x^{\prime} e x y^{\prime} y=x^{\prime} e x$ and, similarly, $y^{\prime} e y x^{\prime} e x=y^{\prime}$ ey. Therefore $(x, y) \in \gamma$ and $\mathscr{L} \subseteq \gamma$. Since $\gamma \subseteq \mathscr{L}$, we get (D).

Assume (D). Let $x \in S$ and $x^{\prime} \in V(x)$. Then $\left(x, x^{\prime} x\right) \in \mathscr{L}$. Since $\mathscr{L}$ is a congruence, for 
all $e \in E$ we have $\left(e x, e x^{\prime} x\right) \in \mathscr{L}$ and hence exex'x=ex. Therefore $e x=e x\left(x^{\prime} x e x^{\prime} x\right)=$ $e x\left(x^{\prime} x e\right)=e x e$. Similarly $e x^{\prime}=e x \cdot e$ and so $(P x)$ is satisfied for all $x \in S$. Thus $S$ is a union of groups. Now (E) follows from [2, Theorem 4.6] and [10]. Clearly (E) implies (A), proving the theorem.

Remark. By the corollary to Theorem 4 , the semilattices $Y$ and $Z$ that occur in Theorem 5 are isomorphic.

In the proof of Theorem 6 below, for any $x \in S, x^{-1}$ denotes the inverse of $x$ in the group $H_{x}$.

THEOREM 6. Let $S$ be a right unipotent semigroup and let $E=E(S)$. Then the following statements are equivalent:

(A) $S$ is a band of groups;

(B) $(Q x)$ is satisfied for all $x$ in $S$;

(C) $\alpha=\gamma=\mathscr{L}$

(D) $\beta=\mathscr{H}=\mathscr{R}$ (equivalently, $\mathscr{R}$ is a congruence on $S$ );

(E) $S$ is a band $E$ of groups.

Proof. Assume (A). Then $a b S=a^{2} b S$ for all $a, b$ in $S$ [1], [2, p. 129]. Let $x \in S$ and $e \in E$. Put $a=x$ and $b=x^{-1} e$. Since $e x^{-1} \in V(x e)$ and $x x^{-1}=x^{-1} x$, from $a b S=a^{2} b S$ we get that $\left(x x^{-1} e, x e x^{-1}\right) \in \mathscr{R}$. Therefore $x x^{-1} e=x e x^{-1}$, giving (B).

Assume (B). Let $x, y \in S, x^{\prime} \in V(x)$ and $y^{\prime} \in V(y)$. Suppose that $(x, y) \in \mathscr{L}$. Then $x y^{\prime} y=x$ and $y x^{\prime} x=y$. Write $a=y^{\prime} y x^{\prime}$. Since $a \in V(x)$ and $x y^{\prime} \in V\left(y x^{\prime}\right)$, for all $e \in E$, using $\left(Q y x^{\prime}\right)$, we have aex $=y^{\prime}\left(y x^{\prime} e x y^{\prime}\right) y=y^{\prime}\left(y x^{\prime} x y^{\prime} e\right) y=y^{\prime} e y$. Therefore $(x, y) \in \alpha$, proving that $\mathscr{L} \subseteq \alpha$. So we get (C).

Assume (C). We first prove that $(Q x)$ is satisfied for all $x \in S$. Let $x \in S, x^{\prime} \in V(x)$ and $e \in E$. Since $\left(x^{\prime}, x x^{\prime}\right) \in \mathscr{L}$, by hypothesis we have $\left(x^{\prime}, x x^{\prime}\right) \in \alpha$; that is, pex' $x^{\prime}$ qexx' for some $p \in V\left(x^{\prime}\right)$ and $q \in V\left(x x^{\prime}\right)$. Premultiplying the equation by $x x^{\prime}$, we get $x x^{\prime} p e x^{\prime}=x x^{\prime} q e x x^{\prime}$. Since $S$ is right unipotent, $x^{\prime} p=x^{\prime} x$ and $x x^{\prime} q=x x^{\prime}$. Therefore $x e x^{\prime}=x x^{\prime} e x x^{\prime}=x x^{\prime} e$, and $(Q x)$ is satisfied for all $x \in S$. This implies that $S$ is a union of groups and $\left(x, x x^{-1}\right) \in \beta$ for all $x \in S$ [12]. Hence

$$
\begin{aligned}
(x, y) \in \beta & \Leftrightarrow\left(x x^{-1}, y y^{-1}\right) \in \beta \\
& \Leftrightarrow x x^{-1}=y y^{-1} \\
& \Leftrightarrow(x, y) \in \mathscr{R} .
\end{aligned}
$$

Since $\beta=\mu \subseteq \mathscr{H}$, we get (D).

Assume (D). Let $x \in S, x^{\prime} \in V(x)$ and $e \in E$. Then, since $\left(x, x x^{\prime}\right) \in \mathscr{R}$, we have $\left(x e, x x^{\prime} e\right) \in \mathscr{R}$. This implies that $x e x^{\prime}=x x^{\prime} e$. So $(Q x)$ is satisfied for all $x \in S$ and hence $S$ is a union of groups. Now by [7, Theorem 4.3] we have $H_{f} H_{g} \subseteq H_{f g}$ for all $f, g \in E$, giving (E). Trivially (E) implies (A). Hence the theorem.

Note added in proof. Statement (2) of Theorem 1 is known. See Tôru Saitô, Note on quasi-inverse semigroups, Semigroup Forum 6 (1973), 129-132. 


\section{REFERENCES}

1. A. H. Clifford, Bands of semigroups, Proc. Amer. Math. Soc. 5 (1954), 499-504.

2. A. H. Clifford and G. B. Preston, The algebraic theory of semigroups, Math. Surveys of the Amer. Math. Soc. 7 (Providence R.I., 1961 (Vol. 1) and 1967 (Vol. 2)).

3. T. E. Hall, On regular semigroups whose idempotents form a subsemigroup, Bull. Austral. Math. Soc. (1969), 195-208.

4. J. M. Howie, The maximum idempotent-separating congruence on an inverse semigroup, Proc. Edinburgh Math. Soc. (2) 14 (1964), 71-79.

5. J. M. Howie, Introduction to semigroup theory (Academic Press, 1976).

6. G. Lallement, Congruences et équivalences de Green sur un demi-groupe régulier, $C . R$. Acad. Sci. Paris Sér. A. 262 (1966), 613-616. 323-341.

7. J. C. Meakin, Congruences on orthodox semigroups, J. Austral. Math. Soc. 12 (1971),

8. W. D. Munn, Fundamental inverse semigroups, Quart. J. Math. Oxford (2) 21 (1970), 157-170.

9. N. R. Reilly and H. E. Scheiblich, Congruences on regular semigroups, Pacific J. Math. 23 (1967), 349-360.

10. P. S. Venkatesan, On decomposition of semigroups with zero, Math. Z. 92 (1966), 164-174.

11. P. S. Venkatesan, Right (left) inverse semigroups, J. Algebra 31 (1974), 209-217.

12. P. S. Venkatesan, On right unipotent semigroups, Pacific J. Math. 63 (1976), 555-561.

13. R. J. Warne, L-unipotent semigroups, Nigerian J. Sci. (2) 5 (1972), 245-248.

14. M. Yamada, On a regular semigroup in which the idempotents form a band. Pacific $J$. Math. 33 (1970), 261-272.

UNIVERSITY OF IBADAN

IBADAN, NigERIA 